\title{
Influência da mobilidade materna na duração da fase ativa do trabalho de parto
}

\author{
Influence of maternal mobility on duration of the active phase of labor
}

Eliane Bioํㅜ, Roberto Eduardo Bittar², Marcelo Zugaib ${ }^{3}$

\begin{abstract}
Resumo
Objetivos: investigar a influência da mobilidade da parturiente durante a fase ativa do trabalho de parto. Métodos: foi realizado ensaio clínico controlado prospectivo, com análise comparativa entre um grupo de tratamento (n=50) e um grupo controle $(\mathrm{n}=50)$, no Centro Obstétrico do Hospital Universitário da Universidade de São Paulo (USP). Os critérios de inclusão foram: primigestas com feto único em apresentação cefálica; idade gestacional entre 37 e 42 semanas; parturientes com pelo menos duas contrações a cada dez minutos e cérvico-dilatação de até $4 \mathrm{~cm}$, além da concordância em assinar o termo de consentimento livre e esclarecido. A evolução para cesárea foi critério de exclusão. Foram acompanhadas por fisioterapeuta durante toda a fase ativa e orientadas a manterem-se em posição vertical e em movimento, de acordo com a fase da dilatação cervical e a descida fetal no canal de parto. O grupo controle teve acompanhamento obstétrico sem a presença do fisioterapeuta. Este grupo foi selecionado retrospectivamente, a partir dos registros de prontuário, seguindo os mesmos critérios de inclusão e exclusão. Resultados: foram acompanhadas 58 primigestas entre 15 e 37 anos. Dessas, 50 mulheres $(86,2 \%)$ evoluíram para parto vaginal, sendo que oito $(13,7 \%)$ evoluíram para cesárea e foram excluídas do estudo. Entre as 50 parturientes acompanhadas, a média de duração da fase ativa foi de cinco horas e 16 minutos, enquanto no grupo controle foi de oito horas e 28 minutos $(\mathrm{p}<0,001)$. Essa diferença se manteve em relação à bolsa amniótica íntegra ou rota. Também quanto ao esvaecimento do colo, apenas no grupo acompanhado as parturientes com colo fino apresentaram, em média, menor tempo de fase ativa $(\mathrm{p}<0,001)$. No grupo acompanhado, nenhuma parturiente fez uso de analgésicos durante a fase ativa contra $62 \%$ do grupo controle $(\mathrm{p}<0,001)$. Neste grupo, todas as parturientes usaram algum tipo de analgesia para o parto; no grupo acompanhado, $76 \%$ das mulheres que usaram anestesia o fizeram entre 9 e $10 \mathrm{~cm}$ de dilatação, sendo que $12 \%$ não fizeram uso de nenhuma anestesia $(p<0,05)$. Não houve diferença significativa entre as médias de peso dos recém-nascidos e os índices de Apgar entre os dois grupos. Conclusões: a mobilidade adequada da parturiente influencia de maneira positiva o trabalho de parto: aumenta a tolerância à dor, evitando o uso de fármacos, e melhora a evolução da dilatação, diminuindo a duração da fase ativa do trabalho de parto.
\end{abstract}

PALAVRAS-CHAVE: Trabalho de parto; Modalidades de fisioterapia; Parto normal; Comportamento materno

\section{Abstract}

Purpose: to investigate the influence of the maternal mobility during the active phase of labor. Methods: a prospective clinical trial was conducted through comparative analysis among a treatment group $(n=50)$ and a control group ( $\mathrm{n}=50$ ), in the Obstetric Center of the Hospital Universitário da Universidade de São Paulo (USP). The inclusion criteria were: primigravidae with a single fetus on cephalic presentation, with 37 to 42 weeks of pregnancy, with two uterine contractions every ten minutes and with cervical dilatation until $4 \mathrm{~cm}$, besides the agreement to sign the free and informed consent term. The evolution of labor for cesarean section was the exclusion criteria. The patients were assisted during the active phase of labor by the physiotherapist and encouraged for staying in vertical position and movement, according to each dilatation stage and fetus head progression. The control group had obstetric support without the presence of the physiotherapist; it was selected retrospectively, according to the same inclusion and exclusion criteria. Results: 58 primigravidae between 15 and 37 years old were accompanied; 50 of them $(86.2 \%)$ evolved to vaginal birth and eight $(13.7 \%)$ evolved to cesarean section and were excluded. Among the patients who were accompanied, the mean of active phase was five hours and 16 minutes, while in the control group it was eight hours and 28 minutes $(\mathrm{p}<0.001)$. This difference was maintained in relation to the amniotic sac either whole or ragged. As for the cervix uterine evanescence, the treatment group showed a smaller period of active phase in association to a thin uterine cervix $(p<0.001)$. In the treatment group, none of the patients used analgesics during the active phase, against $62 \%$ of the control group $(\mathrm{p}<0.001)$. In this group, all the patients used some

Clínica Obstétrica do Hospital das Clínicas da Faculdade de Medicina da Universidade de São Paulo - USP - São Paulo (SP) - Brasil.

1 Fisioterapeuta e Pós-graduanda em Obstetrícia da Faculdade de Medicina da Universidade de São Paulo - USP - São Paulo (SP) - Brasil.

2 Professor Associado do Departamento de Obstetrícia e Ginecologia da Faculdade de Medicina da Universidade de São Paulo - USP - São Paulo (SP) - Brasil.

3 Professor Titular da Disciplina de Obstetrícia da Faculdade de Medicina da Universidade de São Paulo - USP - São Paulo (SP) - Brasil. Correspondência: Eliane Bio

Rua Joaquim Antunes, nº.767, conj. 34 - 05415-012 - São Paulo - SP - Telefone/Fax: 3086-3151 - e-mail: elianebio@uol.com.br 
kind of anesthesia for delivery; in the treatment group, among those who used anesthesia, $76 \%$ did it while the dilatation was 9 or $10 \mathrm{~cm}$ and $12 \%$ did not use any kind of anesthesia $(\mathrm{p}<0.05)$. The average weight of the newborns and the apgar did not show significant difference rates between the two groups. Conclusions: the good performance of maternal mobility has positive influences on the labor process: it increases the tolerance to pain, avoids the use of drugs during labor, improves the evolution of dilatation and reduces the duration of the active phase.

KEYWORDS: Labor, obstetric; Physical therapy modalities; Natural childbirth; Maternal behavior

\section{Introdução}

Na última década, a assistência ao trabalho de parto tem sido motivo de muitas discussões, tanto no que se refere à qualidade propriamente dita, quanto aos procedimentos utilizado ${ }^{1,2}$. O parto hospitalar propicia o contato da parturiente com os inquestionáveis avanços tecnológicos da Obstetrícia para o controle dos riscos materno-fetais, com os recursos farmacológicos para analgesia e anestesia e com os diversos métodos de controle da vitalidade fetal. Entretanto, deixou-se de valorizar orientações e procedimentos simples para o melhor uso do corpo durante o trabalho de parto.

O hábito da mulher de se manter em movimento durante o trabalho de parto e em posição vertical (de pé, sentada e/ou andando) era uma prática comum de quase todas as culturas ${ }^{3}$. Depois que o parto em posição horizontal foi assimilado pela cultura atual, parece que a influência se estendeu também para o trabalho de parto e as parturientes passaram a se manterem deitadas e se movimentarem apenas no leito. Apenas pequena porcentagem de mulheres passou a escolher espontaneamente a deambulação ou outra posição vertical durante o trabalho de parto. Historicamente, as posturas verticais e a movimentação têm sido referidas como eficientes para a evolução da dilatação, para aliviar a dor durante a contração e para facilitar a descida fetal ${ }^{3}$.

Há 30 anos, têm ocorrido esforços em todo o mundo para tentar reintroduzir uma liberdade corporal durante o trabalho de parto. As discussões do gênero pela autonomia e pela participação ativa da parturiente, bem como os argumentos anatomofisiológicos demonstrando a superioridade das posturas verticais em todos os parâmetros da saúde materna e fetal são convincentes, mas insuficientes para uma mudança nas condutas de acompanhamento do trabalho de parto e do parto $^{3,4}$.

A Organização Mundial da Saúde, desde 1996, preconiza, como práticas eficientes para melhorar a evolução do trabalho de parto, a liberdade para a parturiente movimentar-se e não ficar em posição supina ${ }^{5}$.

No Brasil, o Programa de Humanização do Parto e Nascimento do Ministério da Saúde, lançado em junho/2000, formalizou e evidenciou iniciativas que já vinham ocorrendo, no sentido de se recuperar uma participação mais ativa da parturiente em todo o processo do parto. Muitos serviços buscam implementar uma atenção à parturiente menos intervencionista, com práticas baseadas em evidências, menor uso de tecnologias, maior incentivo e ajuda para o parto vaginal $^{4,6,7}$.

Nesse contexto, muitos estudos apontam para a redescoberta das posturas verticais e da mobilidade materna durante o trabalho de parto, como prática que volta a mostrar evidências vantajosas e seguras para a parturiente ${ }^{8}$.

Em nosso meio, não encontramos estudos que investiguem a eficácia das posições verticais e da mobilidade materna durante o trabalho de parto. Portanto, faltam dados objetivos que sustentem orientar tais procedimentos durante a assistência ao parto.

Neste sentido, realizamos um estudo no Hospital Universitário da Universidade de São Paulo (USP), com o objetivo de analisar a influência da mobilidade da parturiente na evolução da fase ativa do trabalho de parto.

\section{Métodos}

O estudo realizado foi um ensaio clínico controlado, prospectivo, com análise comparativa entre um grupo de tratamento e um grupo controle. A população estudada foi de primigestas, admitidas no pré-parto da Divisão da Clínica Obstétrica do Hospital Universitário da USP, no período compreendido entre maio de 2003 e novembro de 2005. O grupo controle teve acompanhamento obstétrico sem a atuação do fisioterapeuta. Este grupo foi selecionado retrospectivamente, a partir dos registros de admissão. 
O cálculo do tamanho da amostra (n) foi de 50 parturientes para cada grupo, para um poder do teste de $80 \%$ e nível de significância de $5 \%$. A estimativa da amostra foi feita visando comparar a média de duração da fase ativa do trabalho de parto entre os dois grupos ${ }^{9}$. Para análise descritiva da caracterização das parturientes estudadas, nas variáveis quantitativas (idade e idade gestacional) foram utilizadas médias e medianas para resumir as informações e desvios padrões mínimo e máximo bem como para indicar a variabilidade dos dados; nas variáveis qualitativas (cor, escolaridade e tipo de parto) foram apresentadas as freqüências relativas e absolutas. Para análise das variáveis de desfecho, foram utilizados: o teste paramétrico $t$ de Student, para comparar a média entre os grupos quanto à duração da fase ativa e para comparar o peso dos recém-nascidos, pois essas variáveis apresentaram distribuição normal. $\mathrm{O}$ teste $\chi^{2}$ de Pearson foi utilizado para medir o uso de analgésicos, enquanto o teste exato de Fisher foi empregado para comparar o uso de anestesia entre os grupos e o uso da anestesia em relação à fase da dilatação. O teste não paramétrico de Mann-Whitney foi utilizado para comparar os indices de Apgar dos recém-nascidos e para comparar a duração da fase ativa em relação à condição da bolsa amniótica e à condição do colo no início do trabalho de parto.

As parturientes foram selecionadas após a admissão no Centro Obstétrico, a partir dos seguintes critérios de inclusão: primigestas em trabalho de parto, com pelo menos duas contrações a cada dez minutos, com dilatação de até $4 \mathrm{~cm}$, com feto único, em apresentação cefálica; idade gestacional entre 37 e 42 semanas confirmada por ultra-sonografia entre a $8^{\mathrm{a}}$ e a $12^{\mathrm{a}}$ semana ou pelo menos duas compativeis, até a $20^{\mathrm{a}}$ semana, com ausência de intercorrências clínicas e obstétricas. A inclusão somente ocorreu após concordância em assinar o termo de consentimento livre e esclarecido. Foi considerado critério de exclusão a evolução para cesárea, por indicação materna e/ou fetal. Os mesmos critérios de inclusão e exclusão foram aplicados na seleção do grupo controle a partir dos registros de prontuário.

O acompanhamento fisioterapêutico foi iniciado após os procedimentos básicos de admissão obstétrica. As parturientes foram acompanhadas durante todo o trabalho de parto até a dilatação total. A evolução da dilatação cervical e da descida do pólo cefálico foi registrada no Partograma de Philpott e Castle ${ }^{10}$. O grau de decida do pólo cefálico foi avaliado pelo método de De Lee e informado ao fisioterapeuta após exame de toque vaginal realizado pelo obstetra.
O método terapêutico priorizou a orientação da mobilidade corporal dirigida para movimentos específicos para o trabalho de parto: posturas verticais (de pé, andando, sentada), movimento articular geral, mobilidade pélvica, relaxamento do períneo, coordenação do diafragma e estímulo da propriocepção.

As orientações fisioterápicas foram feitas de acordo com as exigências de cada periodo funcional do trabalho de parto: dilatação cervical e descida fetal no canal de parto. As posturas e a mobilidade da parturiente foram distintas, durante a contração e nos intervalos destas, levando-se em conta a insinuação, a descida e o desprendimento fetal. Em cada contração, a parturiente experimentava uma nova postura, alternando-as. Nos intervalos entre as contrações, as parturientes mantiveramse em movimento, experimentando uma ou mais posições, enquanto o fisioterapeuta coordenava o movimento e corrigia vícios pélvicos que se evidenciavam durante o movimento, principalmente a orientação articular do sacro.

$\mathrm{Na}$ fase da insinuação do pólo cefálico fetal, as posturas verticais e a mobilidade da parturiente foram orientadas no sentido de ampliar os diâmetros do estreito superior, por meio de pequenos movimentos das articulações lombo-sacra, sacro-ilíacas, coxo-femorais e sinfise púbica ${ }^{11}$. Durante as contrações: correção da lordose lombar, adutores de coxa em distensão ou relaxados com inibição da rotação interna das coxo-femurais; respiração livre sem inspiração ou expiração profundas; estímulo para a percepção do gradiente descendente da contração uterina. Nos intervalos das contrações, as parturientes foram orientadas na mobilidade da seguinte forma: em posição de pé, mobilização articular geral, movendo-se livremente; andar a favor do peso gravitacional, com leve flexão dos joelhos, com a pelve em balaço lateral; respiração livre, mas com incentivo para a expiração.

$\mathrm{Na}$ fase da descida e rotação fetal pelo estreito médio até atingir o estreito inferior da bacia, as parturientes foram orientadas em movimento e posturas verticais, visando aumentar o espaço sacro-púbico, ampliar o diâmetro biciático, afastar os ísquios e ampliar o diâmetro biisquiático, liberar a flexão do cóccix e gerar elasticidade no perineo ${ }^{11}$. Durante as contrações: inibição da contração reativa do períneo, frente à pressão progressiva do pólo cefálico fetal; relaxamento do diafragma pélvico; coxas em abdução e coxo-femurais em rotação externa; diminuir o comando voluntário da força ativa da musculatura profunda do perineo, evitar respiração profunda, relaxar musculatura respiratória acessória. Nos intervalos das contrações, as parturientes foram auxiliadas a manter a mobili- 
dade corporal, alternando posições verticais em pé, sentadas ou andando, mantendo o relaxamento do períneo, coordenando a respiração, abandonando as ordens de contração (conscientes e inconscientes) da musculatura estriada.

A intervenção fisioterapêutica terminou com o final da dilatação cervical. O fisioterapeuta acompanhou a parturiente à sala de parto para não romper bruscamente o vínculo terapêutico estabelecido e integrou-se à equipe obstétrica, estimulando a participação ativa da parturiente no parto.

Incentivaram-se as parturientes a manterem-se em movimento durante todo o trabalho de parto, mas as posturas foram alternadas de acordo com as habilidades motoras individuais $\mathrm{e}$ com a resposta corporal de cada uma. O objetivo de cada procedimento foi explicado à parturiente. Todo trabalho foi dirigido para a ação corporal; a linguagem simples foi intencionalmente usada para dirigir o foco da percepção corporal.
As Figuras 1 e 2 mostram como foi realizada a seqüência de movimentos da parturiente, considerando o ritmo da evolução da dilatação e os períodos sem contração intercalados com as contrações, numa visão de conjunto de como ocorreu a dinâmica corporal ao longo do processo do trabalho de parto.

As parturientes do grupo controle não receberam nenhuma orientação sobre posição ou movimento; não foram impedidas de se movimentarem espontaneamente se desejassem, mas seguiram a orientação habitual do atendimento obstétrico de permanecerem no leito em posição horizontal (supina ou lateral).

O projeto foi aprovado pela Comissão de Ética para Análise de Projetos de Pesquisa do Hospital das Clínicas da Faculdade de Medicina da USP.

Os parâmetros selecionados para análise foram: tipo de parto; tempo de duração da fase ativa do trabalho de parto, dos 3 aos $10 \mathrm{~cm}$ de

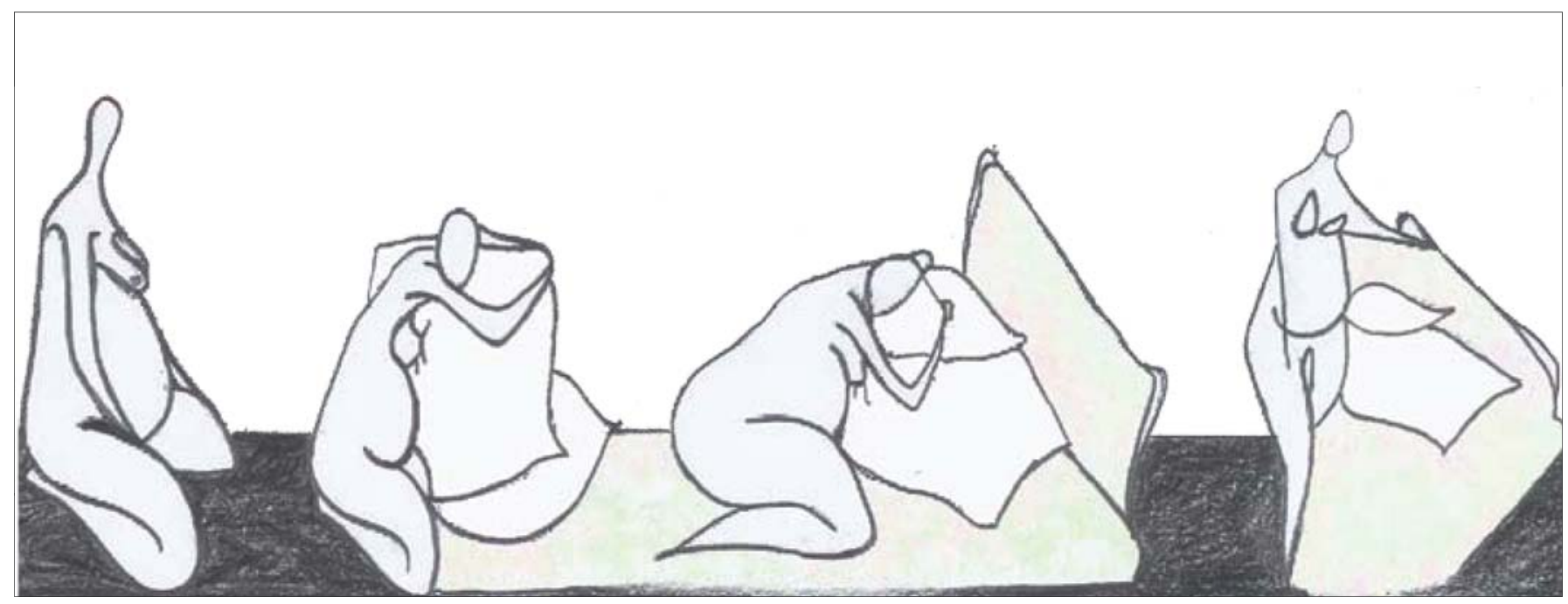

Figura 1 - Posturas verticais realizadas pela parturiente durante as contrações.

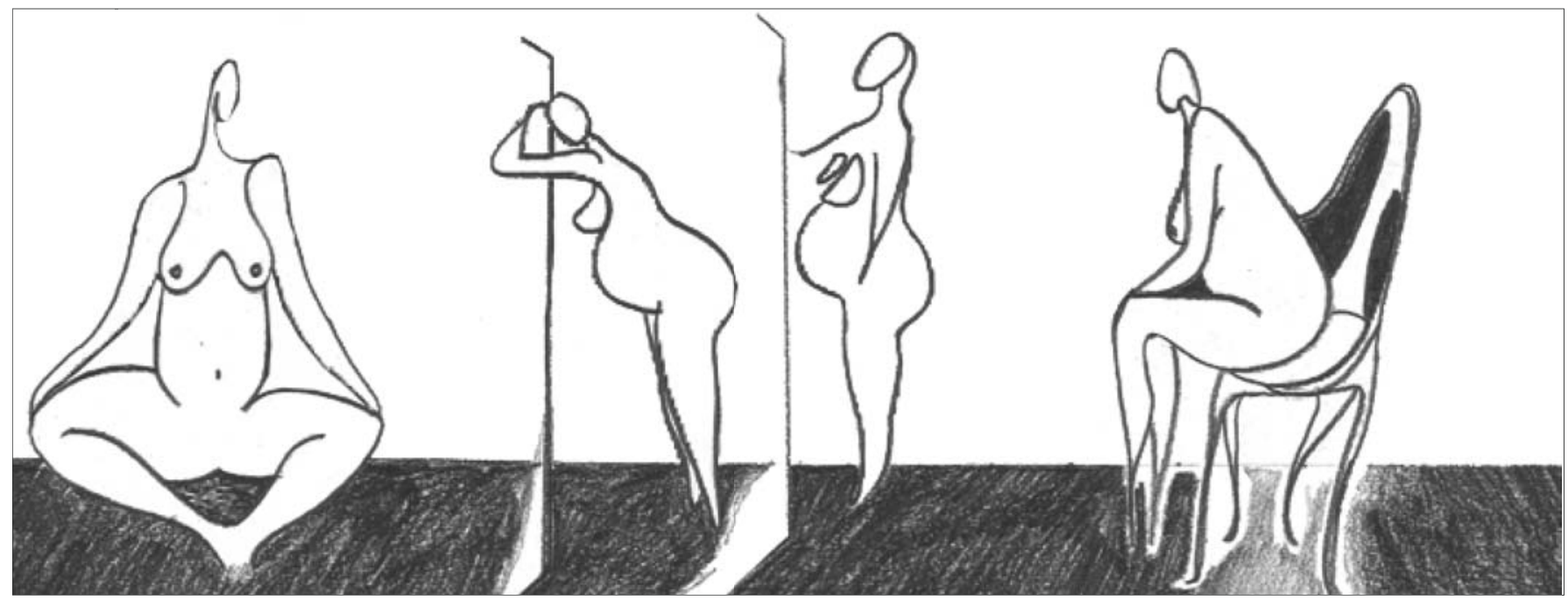

Figura 2 - Posições verticais orientadas à parturiente durante as contrações e movimentos realizados nos intervalos das contrações. 
dilatação; relação entre a duração da fase ativa e a condição da bolsa amniótica (integra ou rota) no início do acompanhamento; relação entre a duração da fase ativa e o esvaecimento do colo uterino (fino, médio ou grosso); uso de analgésicos (meperidina) durante a fase ativa; uso de anestesia (peridural, raqui ou duplo bloqueio) para analgesia de parto; uso de anestesia e nivel da dilatação cervical; peso e índices de Apgar dos recém-nascidos.

\section{Resultados}

Todas as parturientes aceitaram o acompanhamento do fisioterapeuta para se manterem em movimento e em posturas verticais durante o trabalho de parto, isto é, não foi encontrada nenhuma resistência à participação no estudo. Foram acompanhadas 58 primigestas, das quais $50(86,2 \%)$ tiveram parto vaginal e oito $(13,7 \%)$ evoluíram para parto cesárea, sendo excluídas da pesquisa. O grupo controle incluiu somente parturientes com parto vaginal.

A análise estatística da homogeneidade dos grupos (tratamento e controle) com relação às variáveis idade, idade gestacional e cor demonstrou que os grupos foram homogêneos e comparáveis $(p>0,05)$. No grupo tratamento, a média de idade foi de 22 anos $( \pm 5,20)$, mínima de 15 anos e máxima de 35 anos; a mediana da idade gestacional foi de 39 semanas $( \pm 1,10)$; as parturientes foram $67 \%$ de cor branca e $25 \%$ pardas. Quanto ao nivel de escolaridade, 40\% das mulheres tinham grau de instrução elementar: ensino básico incompleto (28\%) ou ensino básico completo (12\%); os outros $60 \%$ apresentavam um grau de instrução mais elevado: ensino fundamental completo (38\%), nivel técnico ou superior. O grupo controle apresentou perfil semelhante: a média de idade foi de 21 anos $( \pm 4,41)$, mínima de 15 anos e máxima de 35 anos; a mediana da idade gestacional foi de 39 semanas $(1,04)$; a maioria era branca (64\%), com $30 \%$ de pardas. Quanto ao nivel de escolaridade, 42\% tinham instrução elementar e 58\%, ensino fundamental completo e nivel superior.

As indicações de cesárea foram devido às seguintes causas: distocia funcional (cinco parturientes), por parada da dilatação cervical e por hipoatividade uterina; desproporção céfalo-pélvica (duas parturientes) e sofrimento fetal intraparto (uma parturiente). Nos casos de falta de maturação cervical (três) e de hipoatividade uterina (dois), os procedimentos fisioterapêuticos tiveram efeito limitado. Essas parturientes apresentavam, em comum, dificuldades na percepção sensorial, um comportamento motor de pouca mobilidade e preferiam a posição horizontal. A intervenção fisioterapêtica foi restrita para as parturientes nas quais a bacia obstétrica era a limitação para o parto vaginal (duas).

Os resultados a seguir referem-se a 100 parturientes: 50 do grupo de tratamento e 50 do grupo controle. Quanto à duração da fase ativa, no grupo tratamento, a média de duração foi de cinco horas e 16 minutos, com variação entre três e nove horas. No grupo controle, a média de duração da fase ativa foi de oito horas e 28 minutos, variando entre quatro e 15 horas (Tabela 1). Esses resultados foram estatisticamente significativos $(\mathrm{p}<0,001)$.

A diferença entre os grupos se manteve, considerando o tempo de fase ativa e a condição da bolsa amniótica (integra ou rota) no início do trabalho de parto: no grupo tratamento, 33 parturientes apresentavam a bolsa integra no início do acompanhamento e tiveram, em média, cinco horas de fase ativa, enquanto no grupo controle as 35 parturientes que apresentavam a bolsa integra tiveram, em média, oito horas e 23 minutos de fase ativa; entre as parturientes que apresentavam bolsa rota no início do acompanhamento, as 17 do grupo tratamento tiveram, em

Tabela 1 - Comparação da duração da fase ativa em relação à bolsa amniótica. Análise separada por grupo.

\begin{tabular}{|c|c|c|c|c|c|c|c|c|}
\hline Grupo & Bolsa amniótica & $\mathrm{n}$ & Média & Mediana & Desvio padrão & Mínimo & Máximo & $p$ \\
\hline & Íntegra & 33 & 5,0 & 5,0 & 1,7 & 3 & 9 & \\
\hline \multirow[t]{3}{*}{ Tratamento } & Rota & 17 & 4,2 & 4,0 & 1,1 & 3 & 7 & 0,184 \\
\hline & Total & 50 & 5,1 & 4,0 & 1,5 & 3 & 9 & \\
\hline & Íntegra & 35 & 8,2 & 8,0 & 2,5 & 4,5 & 15 & \\
\hline \multirow[t]{2}{*}{ Controle } & Rota & 15 & 7,0 & 5,5 & 3,0 & 4 & 14 & 0,116 \\
\hline & Total & 50 & 8,2 & 8,0 & 2,7 & 4 & 15 & \\
\hline
\end{tabular}

Teste não paramétrico de Mann-Whitney. 
média, quatro horas e 29 minutos de fase ativa e as 15 parturientes do grupo controle tiveram, em média, sete horas e sete minutos de duração da fase ativa. A análise foi feita separada por grupo (Tabela 1).

Em relação à duração da fase ativa e esvaecimento do colo, no grupo tratamento, 24 parturientes (48\%) tinham colo fino no início do acompanhamento, $24(48 \%)$ colo médio e duas parturientes $(4 \%)$, colo grosso; no grupo controle, 18 parturientes (36\%) apresentavam colo fino no início do trabalho de parto, $29(58 \%)$ colo médio e três parturientes (6\%), colo grosso. A análise foi feita separadamente por grupo, classificando-se o tipo de colo em fino e médio/grosso. Houve diferença estatisticamente significativa $(\mathrm{p}<0,001)$ na duração da fase ativa e esvaecimento do colo apenas no grupo tratamento: as parturientes com colo fino apresentaram, em média, menor tempo de fase ativa (Tabela 2).

No grupo tratamento, nenhuma parturiente fez uso de analgésicos durante a fase ativa, ao passo que, no grupo controle, 31 parturientes $(62 \%)$ necessitaram de meperidina durante o trabalho de parto. Essa diferença foi estatisticamente significativa $(p<0,001)$.
Em relação ao uso de anestesia para analgesia de parto, houve diferença estatisticamente significativa entre os grupos $(p<0,05)$. No grupo controle, todas as parturientes fizeram uso de algum tipo de anestesia, enquanto no grupo das parturientes acompanhadas, $12 \%$ não fizeram uso de nenhuma anestesia para o parto (Figura 3).

Entre as parturientes que usaram anestesia, também houve diferença entre os grupos em relação à fase da dilatação em que foi usada a anestesia: no grupo controle $40 \%$ das parturientes usaram analgesia para o parto entre 7 ou $8 \mathrm{~cm}$ de dilatação, enquanto que, no grupo-tratamento, $12 \%$ o fizeram nessa fase da dilatação e $76 \%$ entre 9 ou $10 \mathrm{~cm}$ da dilatação (Tabela 3). Essas diferenças foram estatisticamente significativas $(p<0,001)$.

A média do peso dos recém-nascidos foi $3185,90 \mathrm{~kg}$ no grupo de tratamento e $3259,20 \mathrm{~kg}$ no grupo controle, não havendo diferença estatisticamente significativa entre os grupos. Quanto aos índices de Apgar, estes não foram Apgar menor que sete no primeiro minuto, nem no grupo de tratamento nem no controle, não havendo também diferença estaticamente significativa entre eles.

Tabela 2 - Comparação da duração da fase ativa em relação ao colo. Análise separada por grupo.

\begin{tabular}{|c|c|c|c|c|c|c|c|c|}
\hline Grupo & Colo & $\mathrm{n}$ & Média & Mediana & Desvio padrão & Mínimo & Máximo & $p$ \\
\hline & Fino & 24 & 3,7 & 3,7 & 0,7 & 3 & 6 & \\
\hline \multirow[t]{3}{*}{ Tratamento } & Médio/Grosso & 26 & 5,7 & 5,0 & 1,5 & 4 & 9 & $<0,001$ \\
\hline & Total & 50 & 5,1 & 4,0 & 1,5 & 3 & 9 & \\
\hline & Fino & 18 & 7,3 & 6,7 & 2,6 & 4,5 & 15 & \\
\hline \multirow[t]{2}{*}{ Controle } & Médio/Grosso & 32 & 8,1 & 8,2 & 2,7 & 4 & 14 & 0,353 \\
\hline & Total & 50 & 8,2 & 8,0 & 2,7 & 4 & 15 & \\
\hline
\end{tabular}

Teste não paramétrico de Mann-Whitney; $\mathrm{p}<0,05$; Teste exato de Fisher.

Tabela 3 - Comparação entre os grupos no uso de anestesia em relação à fase da dilatação $(\mathrm{em} \mathrm{cm})$.

\begin{tabular}{lccc}
\hline & \multicolumn{2}{c}{ Grupo } & Total \\
& $\begin{array}{c}\text { Tratamento } \\
\mathbf{n}(\%)\end{array}$ & $\begin{array}{c}\text { Controle } \\
\mathbf{n}(\%)\end{array}$ & \\
\hline $7-8 \mathrm{~cm}$ & $6(12,0 \%)$ & $20(40,0 \%)$ & $26(26,0 \%)$ \\
$9-10 \mathrm{~cm}$ & $38(76,0 \%)$ & $30(60,0 \%)$ & $68(68,0 \%)$ \\
Sem anestesia & $6(12,0 \%)$ & & $6(12,0 \%)$ \\
Total & $\mathbf{5 0 ( 1 0 0 , 0 \% )}$ & $\mathbf{5 0 ( 1 0 0 , 0 \% )}$ & $\mathbf{1 0 0 ( 1 0 0 , 0 \% )}$ \\
\hline
\end{tabular}

$\mathrm{p}<0,001$; Teste exato de Fisher.

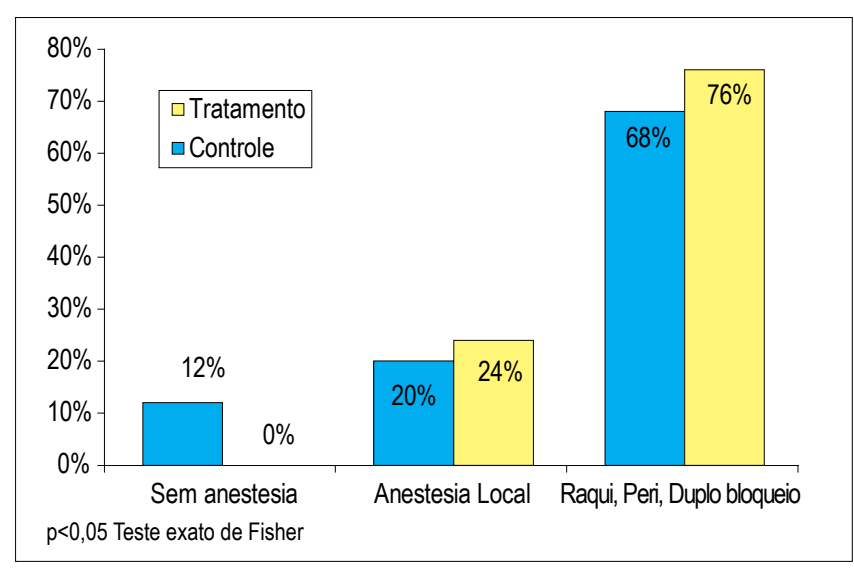

Figura 3 - Distribuição da frequeência do uso de anestesia em cada grupo. 


\section{Discussão}

Na segunda metade do século XX, observaram-se grandes mudanças na assistência ao parto: aumento da medicalização, inúmeras tecnologias e crescentes indicações de parto cesárea ${ }^{1}$. É sabido que a América Latina - especificamente o Brasil - apresenta as mais altas taxas de cesárea entre os países do mundo, apesar de as evidências científicas demonstrarem a vantagem do parto vaginal para a saúde da mulher. Embora admitindo a necessidade do parto cesárea em muitas situações, as elevadas taxas desse tipo de parto entre nós constituem um problema de saúde associado à morbidade materna ${ }^{1}$.

O processo do trabalho de parto, principalmente visando o parto vaginal, é uma tarefa psicomotora para cada mulher. O corpo em movimento ativo é um instrumento que oferece à parturiente um melhor manejo do trabalho de parto, facilitando o parto vaginal. Verificamos, em nosso estudo, que o movimento e a orientação das posições verticais são eficazes para melhorar a evolução da fase ativa e favorecer o parto vaginal, quando comparado ao grupo de parturientes que permaneceram sem mobilidade e em posição horizontal.

A escolha da parturiente de permanecer ativa envolve a interação de fatores fisiológicos, psicológicos e culturais, além de implicar o auxílio da equipe obstétrica para tal. Estudos têm mostrado que a sensação de controle de si mesma, durante o trabalho de parto, traduz-se em melhores resultados materno-fetais: o controle do próprio corpo durante as contrações, a habilidade pessoal para lidar com o medo, o manejo da dor e da condução do processo são fatores que contribuem para a boa experiência e satisfação com o parto e preparação para a maternidade ${ }^{12,13}$. Promover e facilitar a liberdade corporal da mulher durante o trabalho de parto, além de ser prática comprovadamente benéfica, se inclui nas diretrizes dos procedimentos para humanização do parto, no sentido da legitimidade da participação e autonomia da parturiente ${ }^{4}$. Assim, a assistência fisioterapêtica durante o trabalho de parto tem função de favorecer os recursos corporais de cada parturiente e oferecer técnicas e procedimentos terapêticos que resultem numa melhor apropriação de si mesma e do processo do parto, pelo bom uso do corpo.

Alguns estudos em que se empregou a pelvimetria por ressonância magnética (em nãográvidas) contribuíram para a fisiologia obstétrica e demonstraram que as posições verticais influen- ciam a dimensão dos diâmetros pélvicos. Numa população de 35 mulheres, com idade entre 22 e 43 anos, foram feitas imagens da pelve e medidas do conjugado obstétrico, do diâmetro biciático e do diâmetro biisquiático, em posição horizontal e em posição vertical (de joelhos e de cócoras). Os resultados mostraram que: o diâmetro biciático aumenta 6 a $7 \mathrm{~mm}$ nas posições verticais, comparado com a medida em posição horizontal $(\mathrm{p}<0,001)$; o diâmetro biisquiático aumenta $5 \mathrm{~mm}$ em posição de cócoras em relação à posição horizontal ( $\mathrm{p}=0,01)$; o conjugado obstétrico é menor $1 \mathrm{~mm}$ nas posições verticais do que na horizontal $(p=0,01)$. Esses achados mostraram que, nas posições verticais usadas, as dimensões pélvicas se expandiram significativamente, sugerindo vantagens para o trabalho de parto e parto ${ }^{14}$. Essas informações ofereceram aos obstetras a confirmação da mobilidade das articulações da bacia entre si e de que a dimensão da pelve se altera de acordo com a posição adotada. Em estudos clínicos se discute se esses achados e a influência da posição da bacia, associados à pressão do pólo cefálico, à movimentação da parturiente e à posição da mesma durante as contrações são efeitos potenciais para mudança da amplitude pélvica durante o trabalho de parto ${ }^{15}$. Nossos resultados confirmam esses achados, uma vez que, no grupo de parturientes estimuladas a se movimentar em posições verticais, a evolução da dilatação e a descida da apresentação foram mais rápidas do que no grupo que permaneceu sem movimento no leito. A possibilidade de ampliar os diâmetros da bacia, por meio das posições verticais, aliadas aos pequenos movimentos das articulações pélvicas entre si e ao uso da musculatura estriada em coordenação, tiveram um efeito facilitador, diminuindo a duração da fase ativa. A análise recai sobre o espaço pélvico que pode ser criado pela mudança postural quando se quer facilitar o parto vaginal.

O bom uso da musculatura estriada depende da percepção sensorial para a estruturação do movimento coordenado ${ }^{16}$. A falta de percepção sensorial (por dificuldades com o corpo ou por defesas subjetivas) foi um impedimento para manter a parturiente nas posturas verticais e em movimento adequado. As posturas verticais favorecem a percepção do gradiente crescente da contração uterina e ao conseqüente aumento da pressão no períneo; a assistência fisioterapêutica envolveu auxiliá-las a não realizar ação muscular contrária ao processo de dilatação cervical e descida fetal.

A sensação da contração uterina deve migrar da região lombar e baixo-ventre para o períneo, no processo da descida do pólo cefálico durante o 
trabalho de parto. A falta de migração da sensação dolorosa das contrações para o períneo pode ser um sinal indireto de distocia no parto ${ }^{17}$. Pudemos observar o mesmo achado nas parturientes que evoluíram para cesárea por distocia funcional por parada da dilatação.

O manejo da dor exige que o limiar e o limite de tolerância individual sejam avaliados, porém, mais do que ser eliminada, é preciso que a sensação da contração uterina seja um recurso de informação sensorial para estruturar e dirigir a mobilidade da parturiente e organizar o controle voluntário da musculatura estriada. Por isso, foi possivel atuar com essas parturientes, mesmo não tendo sido preparadas durante o pré-natal; as contrações uterinas e as sensações daí decorrentes são recursos de sensibilização para tornar a ação motora funcional para o trabalho de parto.

Ainda hoje se indica o uso de meperidina quando a parturiente manifesta muita dor e desconforto durante as contrações ${ }^{18}$ ou para tentar reduzir a duração do trabalho de parto em parturientes com distocias na fase ativa ${ }^{19}$. Entretanto, no grupo de estudo não houve necessidade de uso de analgésicos. Observamos, assim, que as posturas verticais e o movimento coordenado da pelve, além de melhorarem a evolução da dilatação e da descida da apresentação, tiveram ação na analgesia da dor.

A anestesia peridural, a raquianestesia ou o duplo bloqueio oferecem um efetivo alivio para a dor durante o trabalho de parto e para o período expulsivo, facilitando as condições para o parto vaginal. A revisão sistemática da Biblioteca Cochrane demonstra que o uso da anestesia peridural e duplo bloqueio se associam ao aumento de risco para o parto fórcipe ${ }^{20}$. Em nosso estudo, observamos que o uso do corpo em movimento e na verticalidade evitou ou retardou o uso da anestesia, o que nos possibilitou inferir que a mobilidade da parturiente, especialmente o uso coordenado e consciente da pelve, funcionou como recurso de analgesia. Podemos supor também que fazer uso da anestesia com a dilatação cervical mais adiantada ou no período expulsivo gera mais consciência da região do períneo e permite que o esforço de expulsão seja melhor dirigido.

Ao longo da fase ativa, até a dilatação total, a adaptação osteomuscular da pelve influencia o comportamento do períneo. A pelve óssea assegura a função do períneo desde que suas articulações estejam móveis ${ }^{11}$. Essas possibilidades da fisiologia muscular e articular se demonstraram nas parturientes acompanhadas, na medida em que mantê-las com a mobilidade adequada da pelve nos intervalos das contrações e em posição coordenada durante as contrações inibiu a força contrátil da musculatura do períneo, facilitando a capacidade de elasticidade de suas fibras. Por isso, a descida da apresentação foi mais eficiente e o período de fase ativa menor, em relação ao grupo controle.

Os estudos de eletromiografia de superficie com sensor vaginal, na posição de decúbito dorsal, sentada e em pé, demonstram que a contração dos músculos do assoalho pélvico é menos intensa na posição em pé $^{21}$. A amplitude de contração dos músculos do períneo, sendo menor na posição vertical, responde às exigências da dilatação cervical e elasticidade do períneo necessárias para o parto vaginal. Pudemos notar a importância de manter as coxo-femorais em rotação externa e liberar o diafragma pélvico, para que a parturiente suporte melhor o aumento da pressão do períneo na posição vertical, já que é mais difícil reagir com a contração muscular defensiva. Porém, não basta manter a parturiente na vertical se ela se movimenta com rigidez articular, se não for possivel inibir tensões musculares, vícios pélvicos (especialmente a posição do sacro) ou desvios posturais.

A hipoatividade uterina mostrou ser fator limitante para a eficácia das posições verticais e da mobilidade, como foi observado em duas parturientes que evoluíram para cesárea; avaliase que a movimentação da parturiente auxilia a dinâmica uterina, mas por si só, não gera contração eficiente para dilatação cervical. Assim também, condições imprevistas de sofrimento fetal ou desproporção céfalo-pélvica direcionam condutas obstétricas que se sobrepõem à posição e mobilidade da parturiente, como foi possível verificar em parturientes que tiveram indicação de cesárea.

Compreender os aspectos osteomusculares presentes no mecanismo do trabalho de parto permite melhorar a assistência e facilitar as condições para o parto vaginal. Acreditamos que o maior número de partos vaginais no nosso estudo é resultado dessa ação. Confirma essa nossa análise o fato de não ter ocorrido diferença estatisticamente significativo entre os dois grupos em relação ao peso dos recém-nascidos.

Ainda em relação aos resultados do parto, não foi registrada diferença significativa nos índices de Apgar dos recém-nascidos, o que demonstra as boas condições de nascimento. Podemos discutir apenas que, levando-se em conta a menor duração da fase ativa no grupo acompanhado, essas parturientes chegaram em melhor condição psico-física no período expulsivo. 
Concluímos, portanto, que atuar na estrutura osteomuscular da pelve favorece o mecanismo neuro-hormonal da produção das contrações e evolução da dilatação, que se constitui em prática fisioterapêutica que pode ser usada no trabalho de parto, mesmo quando não houve tal preparação durante a gravidez.

Baseados nos resultados desse estudo, podemos dizer que orientar a postura e a mobilidade adequada à parturiente influencia de maneira positiva a fase ativa do trabalho de parto: aumenta a tolerância da parturiente à dor, evitando o uso de fármacos durante o trabalho de parto e melhora a evolução da dilatação, diminuindo a duração da fase ativa.

\section{Agradecimentos}

Agradecemos à Marcela Giotto Levy, pela criação e execução dos desenhos.

\section{Referências}

1. Cecatti JG, Calderón IMP. Intervenções benéficas durante o parto para a prevenção da mortalidade materna. Rev Bras Ginecol Obstet. 2005;27(6):357-65.

2. Serruya SJ, Cecatti JG, Lago TG. O Programa de Humanização no Pré-natal e Nascimento do Ministério da Saúde no Brasil: resultados iniciais. Cad Saúde Pública. 2004;20(5):1281-9.

3. Racinet C. Positions maternelles pour l'accouchement. Gynecol Obstet Fertil. 2005;33(7-8):533-8.

4. Diniz CSG. Humanização da assistência ao parto no Brasil: os muitos sentidos de um movimento. Ciênc Saúde Coletiva. 2005;10(3):627-37.

5. Care in normal birth: a practical guide. Geneva: World Health Organization; 1996. (WHO/ERH/ MSM/96.24).

6. Serruya SJ, Lago TG, Cecatti JG. O panorama da atenção pré-natal no Brasil e o Programa de Humanização no Pré-natal e Nascimento. Rev Bras Saúde Matern Infant. 2004;4(3):269-79.

7. Domingues RMSM, Santos EM, Leal MC. Aspectos da satisfação das mulheres com a assistência ao parto: contribuição para o debate. Cad Saúde Pública. 2004;20 Supl 1:S52-S62.
8. Rooks JP. Evidence-based practice and its application to childbirth care for low-risk women. J Nurse Midwifery. 1999;44(4):355-69.

9. Armitage P, Berry G. Statistical methods in medical research. 2a ed. Oxford: Blackwell; 1987. p. 179-85.

10.Philpott RH, Castle WM. Cervicographs in the management of labour in primigravidae. I. The alert line for detecting abnormal labour. J Obstet Gynaecol Br Commonw. 1972;79(7):592-8.

11. Calais-Germain B. O períneo feminino. São Paulo: Manole; 2005.

12.Green JM, Baston HA. Feeling in control during labor: concepts, correlates, and consequences. Birth. 2003;30(4):235-47.

13. Spiby H, Slade P, Escott D, Henderson B, Fraser RB. Selected coping strategies in labor: an investigation of women's experiences. Birth. 2003;30(3):189-94.

14. Michel SC, Rake A, Treiber K, Seifert B, Chaoui R, Huch R, et al. MR obstetric pelvimetry: effect of birthing position on pelvic bony dimensions. AJR Am J Roentgenol. 2002;179(4):1063-7.

15. Simkin P. Maternal positions and pelves revisited. Birth. 2003;30(2):130-2.

16. Piret MM, Béziers M. A coordenação motora. São Paulo: Summus; 1994.

17. Sabatino H, Serpa JF, Dias AL, Torresan RZ. Sensação da contração uterina referida durante o trabalho de parto e parto. J Bras Ginecol. 1996;106(11/12): 429-34.

18.Kondo MM. Assistência ao parto. In: Zugaib M, Bittar RE, editores. Protocolos assistenciais: clínica obstétrica da FMUSP. 2a ed. São Paulo: Atheneu; 2003. p. 381-4.

19. Sosa CG, Balaguer E, Alonso JG, Panizza R, Laborde A, Berrondo C. Meperidine for dystocia during the first stage of labor: a randomized controlled trial. Am J Obstet Gynecol. 2004;191(4):1212-8.

20.Anim-Somuah M, Smyth R, Howell C. Epidural versus non-epidural or no analgesia in labour. Cochrane Database Syst Rev. 2006;(1):CD000331.

21.Rett MT, Simões JA, Herrmann V, Marques AA, Morais SS. Existe diferença na contratilidade da musculatura do assoalho pélvico feminino em diversas posições? Rev Bras Ginecol Obstet. 2005;27(1):20-3. 